\title{
Correction to: Telomerase reconstitution contributes to resetting of circadian rhythm in fibroblasts
}

\author{
$\mathrm{Yi} \mathrm{Qu}^{1} \cdot$ Meng Mao ${ }^{1}$ Xihong $\mathrm{Li}^{1} \cdot$ Yanyou $\mathrm{Liu}^{2} \cdot$ Jianmin $\mathrm{Ding}^{3} \cdot$ Zhou Jiang ${ }^{2} \cdot$ Chaomin Wan $^{1} \cdot$ Lin Zhang $^{1}$. \\ Zhengrong $\mathrm{Wang}^{2} \cdot$ Dezhi $\mathrm{Mu}^{1}$
}

Published online: 25 June 2020

(c) Springer Science+Business Media, LLC, part of Springer Nature 2020

\section{Correction to: \\ Molecular and Cellular Biochemistry (2008) 313:11-18 https://doi.org/10.1007/s11010-008-9736-2}

In the original article, Fig. 4b was published incorrectly in which four to five lanes in Pi-ERK and Pi-CREB panels look very similar to each other (Telomerase reconstitution contributes to resetting of circadian rhythm in fibroblasts,
Mol Cell Biochem, 2008, 313:11-18). Since this image was stored in The Experiment Center of the West China Second University Hospital, Sichuan University, which was dissoluted in 2012, the original data cannot be traced. Experiments were therefore redone to verify the result and the correct version of Fig. $4 \mathrm{~b}$ is provided in this correction.

The original article can be found online at https://doi.org/10.1007/ s11010-008-9736-2.

Zhengrong Wang wangzhengrong@126.com

$\triangle$ Dezhi Mu

dezhi.mu@ucsf.edu

1 Department of Pediatrics, West China Second University Hospital, Sichuan University, Chengdu 610041, China

2 Health Ministry Key Lab of Chronobiology, West China Medical Center, Sichuan University, Chengdu 610041, China

3 Department of Physiology, Brody School of Medicine, East Carolina University, Greenville, NC, USA 
B
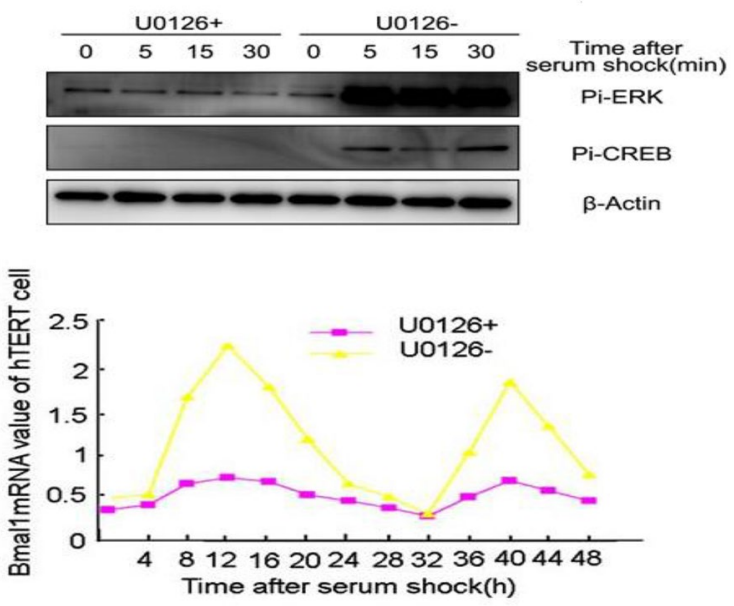

Fig. 4 MAPK cascades activated by serum stimulation. b hTERT fibroblasts were pre-treated with MEK inhibitor U0126, then stimulated with high concentrations of serum, and harvested at the indicated time points. Phospho-ERK and phosphor-CREB were detected by Western blot and BMAL1 mRNA value was detected by RT-PCR as described above. The experiment was repeated for 4 times and the typical result is shown here. It showed that U0126 treatment obviously decreased phospho-CREB value, and the circadian oscillation of BMAL1 was impaired, for the amplitude and peak value of BMAL1 obviously decreased
Publisher's Note Springer Nature remains neutral with regard to jurisdictional claims in published maps and institutional affiliations. 